\title{
Portulaca oleracea Polysaccharides Modulate Intestinal Microflora in Aged Rats in vitro
}

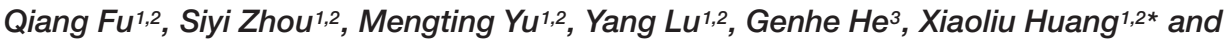 \\ Yushan Huang ${ }^{4 *}$ \\ ' College of Medicine, Jinggangshan University, Ji'an, China, ${ }^{2}$ Ji'an Key Laboratory of Biomedicine, Ji'an, China, ${ }^{3}$ School \\ of Life Sciences, Jinggangshan University, Ji'an, China, ${ }^{4}$ Center for Evidence Based Medical and Clinical Research, First \\ Affiliated Hospital of Gannan Medical University, Ganzhou, China
}

OPEN ACCESS

Edited by:

Fengjie Sun,

Georgia Gwinnett College,

United States

Reviewed by:

Zhaofei Wang,

Shanghai Jiao Tong University, China Xiaolong Yang,

Nantong University, China

${ }^{*}$ Correspondence:

Xiaoliu Huang

huangx11976@163.com

Yushan Huang

3218680@qq.com

Specialty section:

This article was submitted to

Food Microbiology,

a section of the journal

Frontiers in Microbiology

Received: 22 December 2021

Accepted: 13 January 2022

Published: 04 March 2022

Citation:

Fu Q, Zhou S, Yu M, Lu Y, He G,

Huang $X$ and Huang $Y(2022)$

Portulaca oleracea Polysaccharides

Modulate Intestinal Microflora in Aged

Rats in vitro.

Front. Microbiol. 13:841397.

doi: 10.3389/fmicb.2022.841397
To explore the effect of Portulaca oleracea polysaccharides (POP) in regulating intestinal microflora in aged rats in vitro, its intestinal microbial composition was analyzed by $16 \mathrm{~S}$ rDNA high-throughput sequencing, and the level of short-chain fatty acids in fermentation broth was determined by LC-MS. POP significantly upregulated the relative abundance of Lactobacillus, Eggerthella, and Paraprevotella and significantly downregulated Escherichia_Shigella, Bacteroides, and Eubacterium nodatum groups. The $\mathrm{pH}$ value and ammonia nitrogen level decreased significantly in the POP-treated group, resulting in a more short-chain fatty acid consumption which changed the acidbase environment of the fermentation broth. In conclusion, POP is beneficial to aged rats because it can regulate intestinal flora, promote the growth of probiotics, and inhibit the reproduction of pathogenic bacteria.

Keywords: Portulaca oleracea polysaccharides, in vitro fermentation, aged rats, intestinal microflora, probiotics

\section{INTRODUCTION}

The intestinal flora of mammals are colonized by a large number of microflora, whose homeostasis is of great significance to the physiological functions of the organism (Simpson and Campbell, 2015; Zhou et al., 2020). However, with aging, the intestinal flora of the organism also changes, with a decrease in the stability of the dominant species, especially in the elderly population of human beings, thus threatening their health (Salazar et al., 2020). Studies have shown that intestinal flora are closely linked to the development of aging-related diseases, which correlate with reduced intestinal probiotics (Zhou et al., 2020; Chu et al., 2021). In addition, several studies have provided scientific evidence that the abundance of Bifidobacteria decreased, whereas Firmicutes and Enterobacteriaceae increased in the intestines of elderly individuals compared with young individuals (O'Callaghan and van Sinderen, 2016). Studies have also found that the proportions of Bacteroidetes, Firmicutes, and Verrucomicro phyla in the intestinal tract diminished with age (Vemuri et al., 2017; Yu et al., 2017; Lee et al., 2018). The intestinal flora plays an important symbiotic function by producing short-chain fatty acids through fermenting low- or non-digestible carbohydrates that are not easily absorbed by the small intestine (Zhu et al., 2021).

Polysaccharides are complex carbohydrates made up of more than 10 monosaccharide molecules polymerized by glycosidic bonds. In recent years, abundant evidence has demonstrated that polysaccharides can be used as a carbon source of intestinal flora to promote the reproduction of probiotics and consequently regulate intestinal flora through its metabolites, which are vital 
in reshaping the diversity of intestinal microecology, making it a useful prebiotic (Fang et al., 2019). One of the resistant starches, amylose-lipid complex RS5, was reported to be helpful in regulating the microecology of human intestinal flora by promoting the production of butyric acid during in vitro fermentation, where the relative abundance of Bifidobacterium, Dialister, Collinsella, Romboutsia, and Megamonas increased significantly (Qin et al., 2021). In fact, acetic acid, propionic acid, and butyric acid regulate intestinal flora by reducing $\mathrm{pH}$, inhibiting the excessive growth of pathogenic bacteria, such as Escherichia coli, and stimulating the growth of probiotics such as Firmicutes (Ghosh et al., 2011; Zimmer et al., 2012). Shortchain fatty acid production is considered a marker of interactions between host and intestinal flora, which also uncovers the causality between accelerated aging and flora dynamics in elderly individuals (Mangiola et al., 2018).

Purslane, a medicinal plant, is widely used in Traditional Chinese Medicine (Zhou et al., 2015). As a common homology of medicine and food, it also has a variety of indications (Iranshahy et al., 2017). Purslane is rich in bioactive substances, multiple flavonoids, alkaloids, polysaccharides, fatty acids, terpenoids, steroids, and proteins and is high in vitamins and minerals (Hu et al., 2018; Miao et al., 2019). Polysaccharides are the main active ingredients that have been widely studied. Results of the structural analysis show that glucose and galactose are the main monosaccharide molecules of Portulaca oleracea polysaccharide (POP). Research has demonstrated that POP exhibits antitumor and antimicrobial activities, improves diabetes, and promotes oxidation resistance (Dong et al., 2010; $\mathrm{Hu}$ et al., 2018). Our recently published study revealed that POP significantly improved the gut microbiota composition of weaned rats and promoted colonization probiotics such as Lactobacilli and Bifidobacteria, thus affecting the metabolic function of rats (Huang et al., 2021). This suggests that POP may play a role as a prebiotic in promoting the reproduction of intestinal probiotics and modulating the diversity of intestinal microecology. However, there are limited studies on the effect of POP as a prebiotic in gut microbiota homeostasis of aging individuals. The in vitro fermentation model is also considered to be an effective tool for investigating colon microflora in a highly controlled environment. Therefore, in the present study, based on our former results, we explored the effect of POP on regulating intestinal microflora of elderly rats in vitro, which provides potential insight into the development and utilization of POP as food.

\section{MATERIALS AND METHODS}

\section{Reagents}

Purslane polysaccharides $(\geq 50 \%)$ are purchased from Lanzhou Wotelaisi Biotechnology Co., Ltd. (Wotls, Lanzhou, China). Methanol, distilled deionized water, formic acid, and acetonitrile (HPLC grade) were purchased from Thermo Fisher Scientific (Waltham, MA, United States); 3-NPH, EDC, and standard products (HPLC grade) were purchased from Sigma-Aldrich (St. Louis, MO, United States). Related analytical reagents were purchased from Sinopharm Chemical Reagent Co., Ltd (Shanghai, China). The basic medium was prepared as the methodology reported by Mandalari et al. (2008) with minor modifications (2 g/l peptone, $2 \mathrm{~g} / \mathrm{l}$ yeast extract, $0.1 \mathrm{~g} / \mathrm{l} \mathrm{NaCl}$, $0.04 \mathrm{~g} / \mathrm{l} \mathrm{K} \mathrm{HPO}_{4}, 0.04 \mathrm{~g} / \mathrm{KH}_{2} \mathrm{PO}_{4}, 0.01 \mathrm{~g} / \mathrm{l} \mathrm{MgSO}_{4} \cdot 7 \mathrm{H}_{2} \mathrm{O}$, $0.01 \mathrm{~g} / \mathrm{l} \mathrm{CaCl} \cdot 6 \mathrm{H}_{2} \mathrm{O}, 2 \mathrm{~g} / 1 \mathrm{NaHCO}_{3}, 2 \mathrm{ml}$ Tween 80, $0.02 \mathrm{~g} / \mathrm{l}$ hemin, $10 \mu \mathrm{l}$ vitamin $\mathrm{K}_{1}, 0.5 \mathrm{~g} / \mathrm{l}$ cysteine $\mathrm{HCl}$, and $0.5 \mathrm{~g} / \mathrm{l}$ bile salts, $\mathrm{pH} 7.0)$.

\section{In vitro Fermentation of Purslane}

Fecal samples collected by aseptic manipulation from five healthy Sprague-Dawley (SD) aged (21-month-old) rats were pooled with equal amounts and immediately placed inside an anaerobic chamber. $5 \mathrm{~g}$ of fecal samples was taken, and 20 times of the fecal weight of sterile saline was added to the anaerobic chamber, and the mixtures were fully shaken. The mixtures were filtered with eight layers of sterile gauze and transferred into another sterile sample bottle and then seeded into the basic medium to a final volume of 11 for bacteria fermentation. The experiments were divided into six groups: Con1 (control group, fermented for $24 \mathrm{~h}$ ), Con2 (control group, fermented for $48 \mathrm{~h}$ ), LPOP1 (low-dose group of purslane polysaccharide fermented for $24 \mathrm{~h}$ ), LPOP2 (low-dose group of purslane polysaccharide fermented for $48 \mathrm{~h}$ ), HPOP1 (high-dose group of purslane polysaccharide fermented for $24 \mathrm{~h}$ ), and HPOP2 (high-dose group of purslane polysaccharide fermented for $48 \mathrm{~h}$ ). The fermentation volume of all groups was in $10 \mathrm{ml}$ fermentation solution, the Con groups contained no POP, the LPOP groups contained POP $(0.02 \mathrm{~g} / \mathrm{ml})$, and the HPOP groups contained POP $(0.03 \mathrm{~g} / \mathrm{ml})$. Each group was repeated with five independent experiments. Gas production of the anaerobic cultures was recorded at $0,12,24,36$, and $48 \mathrm{~h}$ post inoculation (hpi), and the fermentation culture was collected at 24 and 48 hpi. Supernatants of the culture were separated by centrifugation for detection of $\mathrm{pH}$, ammonia nitrogen, and shortchain fatty acid levels. The precipitates were stored at $-80^{\circ} \mathrm{C}$ for further DNA extraction.

\section{Analysis of Ammonia}

For ammonia analysis, $2.5 \mathrm{ml}$ phenol chromogenic agent was added into a $10-\mathrm{ml}$ tube, and $10 \mu \mathrm{l}$ fermentation supernatants was added later. Then, $2.0 \mathrm{ml}$ hypochlorite solution was also added into the tube. After shaking and mixing, the tube was incubated in water bath at $40^{\circ} \mathrm{C}$ for $15 \mathrm{~min}$ then cooled to room temperature to measure the absorbance value $\left(\mathrm{OD}_{625}\right)$, and the ammonia content was calculated according to the standard curve.

\section{Determination of Short-Chain Fatty Acids}

Short-chain fatty acids were analyzed by LC-MS/MS. A volume of $100 \mu \mathrm{l}$ supernatant was added with $100 \mu \mathrm{l} 50 \%$ acetonitrileaqueous solution $(\mathrm{v} / \mathrm{v})$, and then grinding for $3 \mathrm{~min}$. After that, the samples were sonicated in an ice water bath for $10 \mathrm{~min}$ and then centrifuged for $10 \mathrm{~min}$, at $4^{\circ} \mathrm{C}, 12,000 \mathrm{rpm}$, followed by sample derivation and stored at $-80^{\circ} \mathrm{C}$ for further analysis. The 5- $\mu$ l samples were added into a high-performance liquid chromatograph (Nexera UHPLC LC-30A, Shimazin, Japan) with a color matching column (ACQUITY UPLC BEH C18 $100 \mathrm{~m} \times 2.1 \mathrm{~mm} \times 1.7 \mu \mathrm{m}$, Waters) and a high-resolution mass 
spectrometer (AB SCIEX QTRAP 5500, AB SCIEX). The mass spectrum conditions are as follows: $0.1 \%$ formic acid-aqueous solution (A) and acetonitrile (B) are used as mobile phase, mass flow rate is $0.35 \mathrm{ml} / \mathrm{min}$; curtain gas: 35 (psi); collision-activated dissociation (CAD) parameters: medium; negative ion spray voltage: $-4,500(\mathrm{~V})$; ion source temperature: $450\left({ }^{\circ} \mathrm{C}\right)$; column temperature: $40\left({ }^{\circ} \mathrm{C}\right)$, ion source: gas 1:50 (psi); gas 2:60 (psi). Various short-chain fatty acids were determined according to the retention time, and the concentrations were calculated based on the standard curve.

\section{DNA Extraction and Gene Amplification}

Cecal content samples of the rats were collected and quickfrozen, then stored at $-80^{\circ} \mathrm{C}$ for further analysis. Total genomic DNA was extracted using the DNA Extraction Kit (Magen, Guangzhou, China) according to the manufacturer's instructions. The concentration of DNA was verified with a NanoDrop 2000 spectrophotometer (Thermo Fisher Scientific, Waltham, MA, United States) and agarose gel electrophoresis, respectively. All DNA samples were stored at $-20^{\circ} \mathrm{C}$ for further study. PCR amplification of the V3-V4 hypervariable regions of the bacterial $16 \mathrm{~S}$ rRNA gene was carried out with a $25-\mu \mathrm{l}$ reaction mixture using universal primers (343F: 5'-TACGGRAGGCAGCAG3'; 798R: 5'-AGGGTATCTAATCCT-3'). The reverse primer contained a sample barcode, and both primers were connected with an Illumina sequencing adapter.

\section{Library Construction and Sequencing}

The amplicon quality was visualized by gel electrophoresis. The PCR products were purified with Agencourt AMPure XP beads (Beckman Coulter Co., Brea, CA, United States) and quantified using the Qubit dsDNA assay kit. The concentrations were then adjusted for sequencing. Sequencing was performed on an Illumina NovaSeq 6000 with two paired-end read cycles of 250 bases each (Illumina Inc., San Diego, CA, United States; Oe Biotech Company, Shanghai, China).

\section{Bioinformatic Analysis}

Paired-end reads were preprocessed with Trimmomatic software (Bolger et al., 2014) to detect and cut off ambiguous bases (N). It also cut off low-quality sequences with an average quality score below 20 using a sliding window trimming approach. After trimming, paired-end reads were assembled using FLASH software (Reyon et al., 2012). Parameters of assembly were $10 \mathrm{bp}$ of minimal overlapping, $200 \mathrm{bp}$ of maximum overlapping, and $20 \%$ of maximum mismatch rate. Further denoising of sequences was performed as follows: reads with ambiguous, homologous sequences or below 200 bp were abandoned. Reads with 75\% of bases above Q20 were retained by QIIME software (version 1.8.0) (Caporaso et al., 2010). After that, reads with chimera were detected and removed using VSEARCH (Rognes et al., 2016). Clean reads were subjected to primer sequence removal and clustering to generate operational taxonomic units (OTUs) using VSEARCH software with a $97 \%$ similarity cutoff (Rognes et al., 2016). The representative read of each OTU was selected using the QIIME package. All representative reads were annotated and blasted against Silva database (Version 132) using the RDP classifier (confidence threshold was 70\%) (Wang et al., 2007). The microbial diversity in cecal content samples was estimated using the alpha diversity that includes Chaol index, Shannon index, Observed-species, Simpson, Goods-coverage, and PDwhole-tree. The UniFrac distance matrix performed by QIIME software was used for unweighted UniFrac principal coordinate analysis (PCoA) and phylogenetic tree construction. The $16 \mathrm{~S}$ rRNA gene amplicon sequencing and analysis were conducted by Oe Biotech Co., Ltd. (Shanghai, China).

\section{Statistical Analysis}

Experimental data in the study are reported as mean \pm standard deviation (SD) of at least biological duplicates. One-way analysis of variance (ANOVA) and Duncan's multiple-range tests were employed to analyze significant differences in gas production, $\mathrm{NH}_{3}-\mathrm{N}$ value, $\mathrm{pH}$ value, short-chain fatty acid (SCFA), and $\alpha$-diversity $(p<0.05)$ between groups using SPSS version 19.0 (SPSS Inc., Chicago, IL, United States). Gas, $\mathrm{NH}_{3}-\mathrm{N}, \mathrm{pH}$, and SCFA profiles were plotted in the GraphPad Prism version 8.0 (GraphPad Software, Inc., La Jolla, CA, United States).

\section{RESULTS}

\section{Gas Production, $\mathrm{NH}_{3}-\mathrm{N}$ Value, and $\mathrm{pH}$ Value Analysis}

As fermentation time increased, gas production in the HPOP and LPOP groups changed significantly compared with that in the Con group. Moreover, gas production in the HPOP group was significantly higher than in the LPOP and Con groups (Figure 1A). At the 24- and 48-h fermentation points, both ammonia nitrogen $\left(\mathrm{NH}_{3}-\mathrm{N}\right)$ and $\mathrm{pH}$ values were significantly higher in the Con group than in the HPOP and LPOP groups (Figures 1B,C).

The results also showed that POP inhibited the production of short-chain fatty acids in the fermentation broth. After 24 and $48 \mathrm{~h}$ of fermentation, the concentrations of acetic acid (Figure 2A), butyric acid (Figure 2B), propionic acid (Figure 2C), and isovalerate (Figure 2D) in the fermentation broth of POP groups (both low- and high-dose groups) significantly decreased compared with Con groups; however, there was no difference between the LPOP and HPOP groups (Figure 2).

\section{Effects of Portulaca oleracea Polysaccharides on Microbial Community Distribution}

The Good's coverage indices in the six groups were all greater than $99 \%$, which indicated that most of the bacteria present in the samples were identified (Table 1). Purslane polysaccharides were found to significantly decrease fecal microbiota richness (Shannon, Simpson, and PD-whole-tree) and diversity (Chaol and observed-species) after 24 or $48 \mathrm{~h}$ of in vitro fermentation compared to the control groups (Table 1). In addition, POP altered bacterial $\beta$-diversity (Figure 3 ). Principal component analysis (PCA) revealed the distinct clustering of 
A

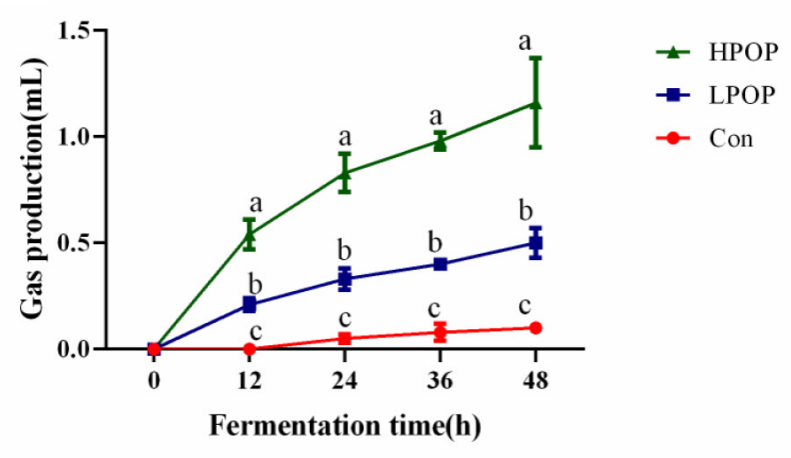

C
B

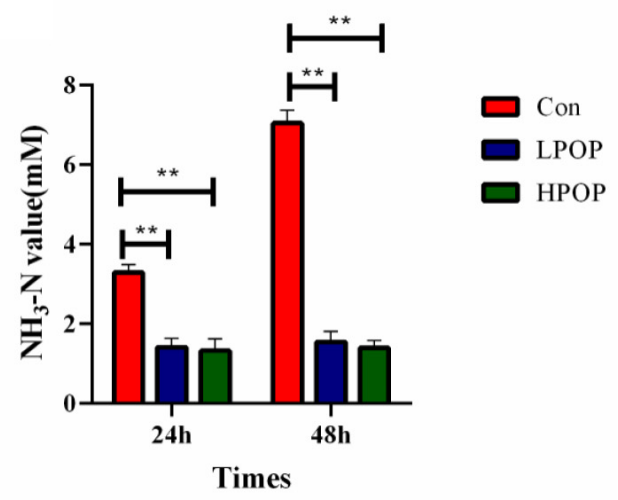

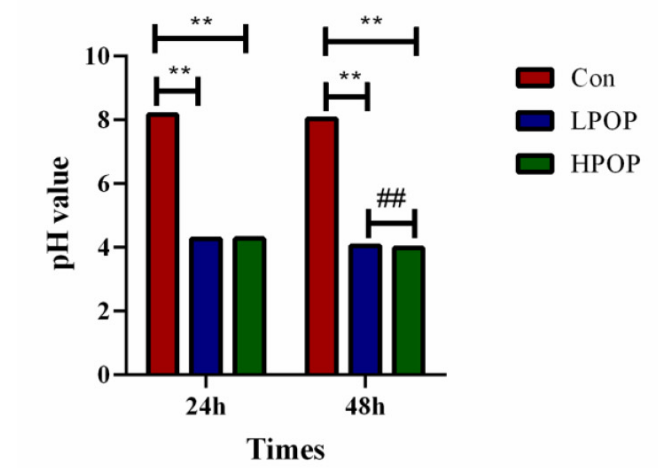

FIGURE 1 | Gas production of the anaerobic cultures was recorded at 0, 12, 24, 36, and $48 \mathrm{~h}$ post inoculation (hpi), and the fermentation culture was collected at 24 and 48 hpi. Supernatants of the culture were separated by centrifugation for detection of pH and ammonia nitrogen levels. Gas production, $\mathrm{NH}_{3}-\mathrm{N}$, and $\mathrm{pH}$ value during $48 \mathrm{~h}$ in vitro fecal fermentation with POP. (A) Gas production. (B) $\mathrm{NH}_{3}-\mathrm{N} .{ }^{* \star} P<0.01$ and ${ }^{\# \#} P<0.01$ vs different groups, respectively. (C) pH value.

microbiota compositions for the six groups. The microbiota compositions of LPOP1, LPOP2, HPOP1, and HPOP2 were not comparable; however, Con1 and Con2 were significantly comparable, but Con groups differed greatly from POP groups in the PC1 analysis, which was $16.1 \%$ of the total variation (Figure 3).

\section{Microbial Composition at Phylum and Genus Levels}

Microflora in fermentation cultures of different groups are mainly composed of Firmicutes, Bacteroidetes, Proteobacteria, and Actinobacteriota when categorized at the phylum level. Nine and six different phyla were observed with ANOVA analysis after 24 and $48 \mathrm{~h}$ of fermentation, respectively. Compared with the Con1 group, the abundance of Firmicutes in the LPOP1 and HPOP1 groups was significantly upregulated, while Bacteroidetes, Proteobacteria, Fusobacteriota, Desulfobacterota, Actinobacteriota, Acidobacteriota, Cyanobacteria, and others showed a significant decrease (Figure 4A). In addition, compared with the Con2 group, the relative abundance of Firmicutes in the LPOP2 and HPOP2 groups was significantly upregulated, whereas the relative abundance of Proteobacteria, Desulfobacterota, Bacteroidota, Fusobacteriota, and others was significantly decreased (Figure 5A).
At the genus level, Escherichia-Shigella and Lactobacillus were dominant in the Con and POP groups, respectively. After fermentation for 24 and $48 \mathrm{~h}$, ANOVA analysis showed that 87 and 78 different bacterial genera were observed, respectively. Compared with the Con1 group, the relative abundance of Lactobacillus in the LPOP1 and HPOP1 groups increased significantly. However, other genera of microflora such as Escherichia-Shigella, Parasutterella, and Klebsiella decreased significantly (Figure 4B). Compared with the Con2 group, the relative abundance of Lactobacillus in the LPOP2 and HPOP2 groups was much higher. Conversely, the relative abundance of Escherichia-Shigella, Parasutterella, Phascolarctobacterium, Eubacterium nodatum_group, and Klebsiella decreased significantly (Figure 5B).

\section{Screening of Different Species Between Groups}

To identify specific microflora associated with POP, the linear discriminant analysis effect size (LEfSe) method was used to compare the differences in microflora between the Con and POP-treated groups after fermentation. The cladding diagrams of fermentative microorganisms and dominant bacteria composition are presented in Figures 6B, 7B. The most differentially abundant taxa are shown in Figures 6A, 7A. 
A

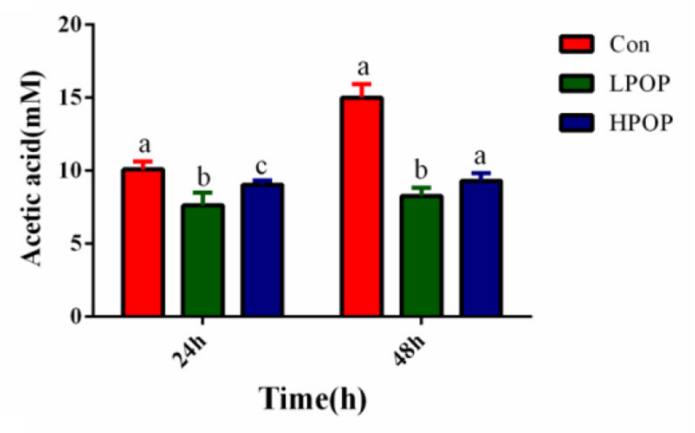

C

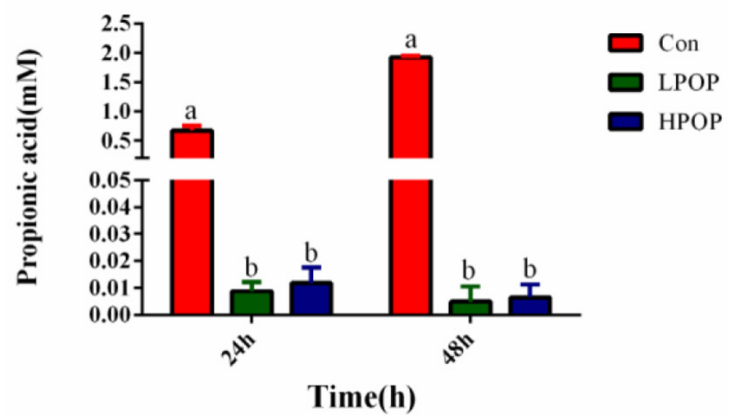

B
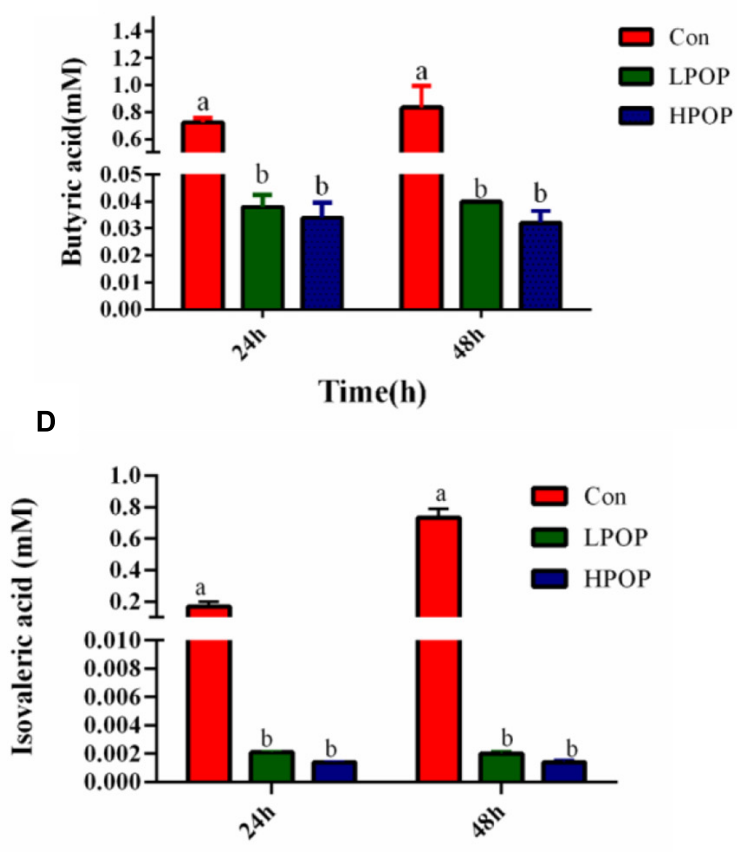

$\operatorname{Time}(\mathbf{h})$

FIGURE 2 | Acetic acid (A), butyric acid (B), propionic acid (C), and isovaleric acid (D) production after 24 and $48 \mathrm{~h}$ in vitro fecal fermentation of POP.

TABLE 1 | $\alpha$-Diversity after 24 and $48 \mathrm{~h}$ in vitro fecal fermentation of POP.

\begin{tabular}{|c|c|c|c|c|c|c|}
\hline Sample & Chao1 & Observed-species & Shannon & Simpson & Goods-coverage & PD-whole-tree \\
\hline Con1 & $762.89 \pm 16.74^{a}$ & $614.36 \pm 25.81^{a}$ & $3.21 \pm 0.31^{a}$ & $0.668 \pm 0.074^{b}$ & $0.9968 \pm 0.0001^{a}$ & $24.23 \pm 0.84^{a}$ \\
\hline LPOP1 & $576.20 \pm 41.15^{b}$ & $428.04 \pm 36.33^{b}$ & $2.68 \pm 0.11^{\mathrm{b}}$ & $0.679 \pm 0.042^{b}$ & $0.9973 \pm 0.0002^{b}$ & $18.62 \pm 1.56^{b}$ \\
\hline HPOP1 & $538.11 \pm 18.94^{b}$ & $370.18 \pm 40.07^{c}$ & $3.06 \pm 0.42^{a b}$ & $0.778 \pm 0.070^{a}$ & $0.9975 \pm 0.0001^{b}$ & $16.51 \pm 1.54^{c}$ \\
\hline Con2 & $765.34 \pm 45.08$ & $620.08 \pm 42.26$ & $3.59 \pm 0.12$ & $0.711 \pm 0.033$ & $0.9971 \pm 0.0001$ & $23.97 \pm 1.72$ \\
\hline LPOP2 & $528.94 \pm 64.93^{\star}$ & $382.30 \pm 48.16^{\star}$ & $2.57 \pm 0.11^{*}$ & $0.686 \pm 0.012$ & $0.9976 \pm 0.0004^{*}$ & $16.82 \pm 1.96^{\star}$ \\
\hline HPOP2 & $529.33 \pm 41.76^{\star}$ & $371.58 \pm 35.76^{\star}$ & $2.64 \pm 0.44^{\star}$ & $0.705 \pm 0.072$ & $0.9976 \pm 0.0002^{*}$ & $16.47 \pm 1.55^{\star}$ \\
\hline
\end{tabular}

Values are mean $\pm S D$.

Significant $(p<0.05)$ differences among $24 \mathrm{~h}$ are indicated with different letters, ${ }^{*} p<0.05$ means $v$ Con2.

After $24 \mathrm{~h}$ of fermentation, the relative abundance of Bacilli-Lactobacillus, Eggerthella, and Firmicutes in the LPOP1 group was significantly increased, and in the HPOP1 group, the relative abundance of other bacteria was also significantly increased. In addition, the Escherichia-Shigella, Bacteroides, Clostridium sensu stricto 13, Enterobacter, Intestinimonas, Gardnerella, Proteus, Ruminococcus torques group, Eubacterium nodatum group, Prevotella, Clostridium sensu stricto 18, Caldibacillus, and Eisenbergiella in the Con 1 group showed a significant increasing trend (Figure 6A). Furthermore, when the fermentation time was extended to $48 \mathrm{~h}$, compared with the Con group, the relative abundance of Bacilli-Lactobacillus and Firmicutes in the LPOP2 group and Paraprevotella in the HPOP2 group showed a significant increasing trend. In the Con2 group, the relative abundance of several other phyla (i.e., EscherichiaShigella, Bacteroides, Parasutterella, Enterobacter, Veillonella, Clostridium sensu stricto 13, Marinobacter, Parabacteroides,
Erysipelotrichaceae UCG-003, Ruminococcus_torques_group, Tyzzerella, Mobiluncus, Gardnerella, and Clostridium sensu stricto 18) also significantly increased (Figure 7A).

\section{Functional Properties Inherent to the Microbiomes Using Phylogenetic Investigation of Communities by Reconstruction of Unobserved States}

Phylogenetic Investigation of Communities by Reconstruction of Unobserved States (PICRUSt) is designed to predict metagenome functional content from marker gene surveys and full genomes. The marker gene of $16 \mathrm{~s}$ rRNA was used to amplify the subsequence to estimate the metabolomic function spectrum of a microbial community. According to the PICRUSt results, there were significant differences in metabolic potential between the Con and POP groups (Figure 8). 


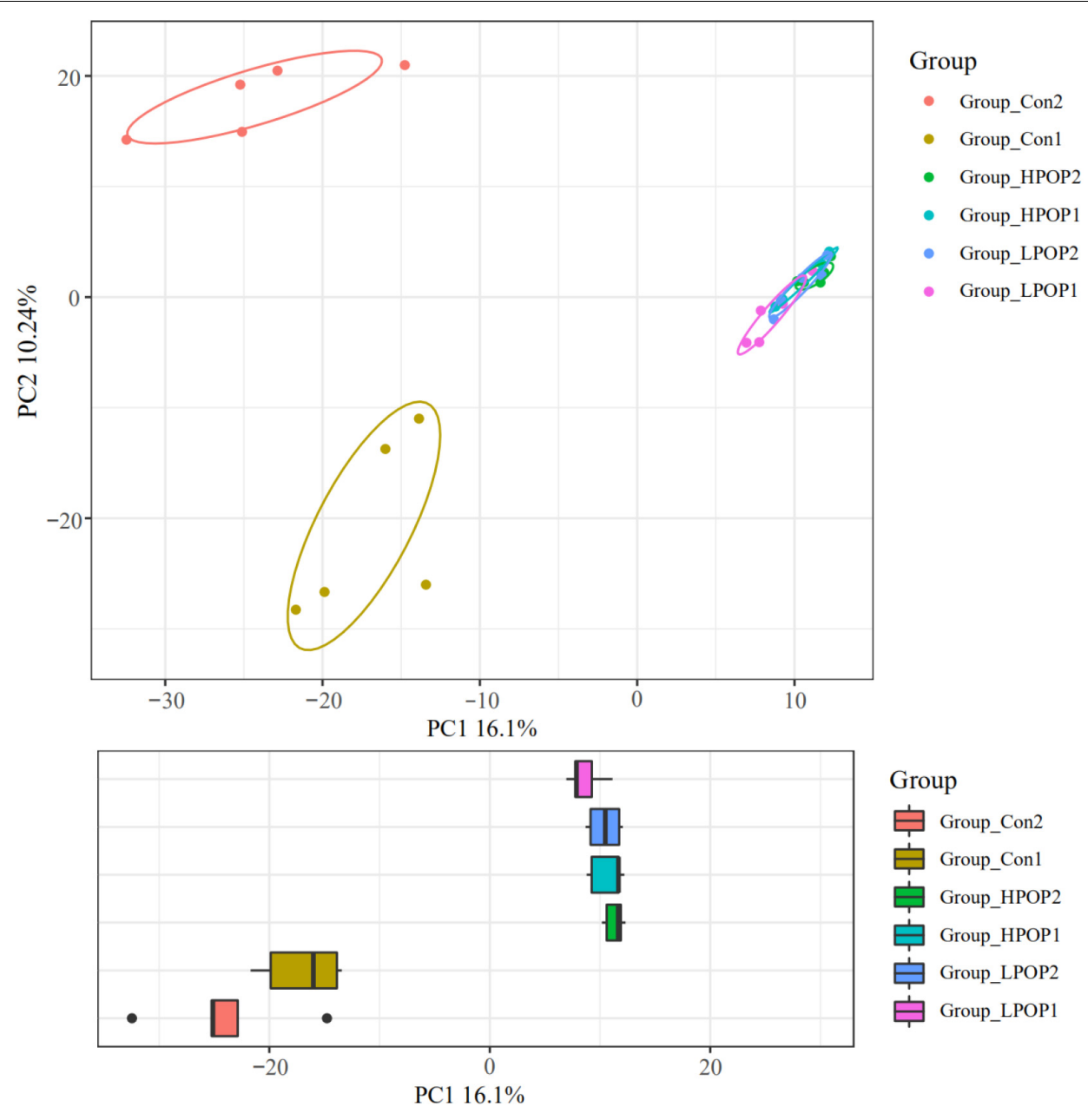

FIGURE 3 | Principal component analysis (PCA) of the microbial community composition after 24 and $48 \mathrm{~h}$ in vitro fecal fermentation with POP.

Compared with the POP-treated group, the Con groups showed an increasing trend in the expression of genes associated with environmental adaptation, xenobiotics biodegradation and metabolism, metabolism of cofactors and vitamins, metabolism of terpenoids and polyketides, lipid metabolism, metabolism of amino acids, carbohydrate metabolism, glycan biosynthesis and metabolism, energy metabolism, folding, sorting and degradation, transport and catabolism, cellular processes, signaling and metabolism, membrane transport, cell motility, biosynthesis of other secondary metabolites, cancers, metabolic diseases, cardiovascular diseases, neurodegenerative diseases, infectious diseases, circulatory system, digestive system, immune system, endocrine system, enzyme families, poorly characterized transcription, signal transduction, and genetic information processing. Conversely, the expression of genes involved in cell growth and death, translation, immune system diseases, signaling molecules, and interaction decreased.

Regarding fermentation time, in both LPOP1 and LPOP2 groups, genes of replication and repair, nucleotide metabolism, excretory system, nervous system, cell growth and death, translation, immune system diseases, xenobiotics biodegradation and metabolism, metabolism of cofactors, cell communication, and sensory system showed a noticeable change in expression level. The expression of genes for environmental adaptation, replication, and repair in the HPOP1 and HPOP2 groups was also significantly changed.

\section{DISCUSSION}

The gut microbiome plays a crucial role in human health (Gomaa, 2020). There is increasing evidence that natural products and their bioactive compounds have aroused interest in their diverse bioactivities (Kim and Kim, 2018; Xu et al., 2018). Polysaccharides are functional natural products that have been widely studied. Studies have shown that polysaccharides that are hard to digest can improve sugar metabolism and prevent diseases by altering the composition of the gut microbiome (Wang H. et al., 2018; Wang M. et al., 2018). Our results demonstrate that the $\mathrm{pH}$ values of fermentation cultures in the LPOP and HPOP groups are significantly lower than those in the Con group at 24 and $48 \mathrm{~h}$ of fermentation. The alteration in the 
A

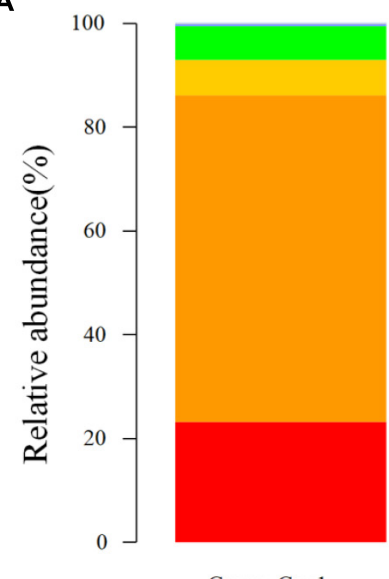

Group_Conl

- Firmicutes - Proteobacteria Actinobacteriota - Bacteroidota Fusobacteriota - Desulfobacterot - Acidobacteriota - Campilobacterota

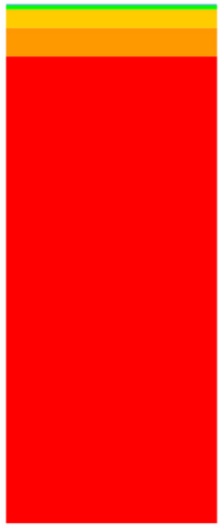

Group_HPOP1

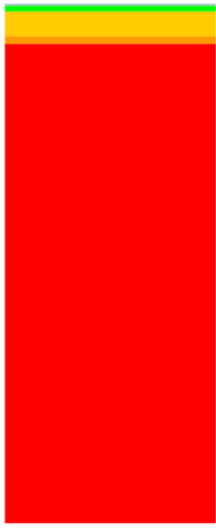

Group_LPOP1

- Cyanobacteria

- Myxococcota

Gemmatimonadota

Nitrospirota

Spirochaetota

- RCP2-54

- Caldisericota

others

B

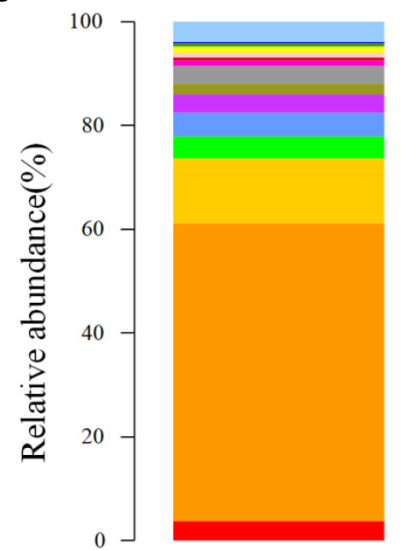

Group_Con1

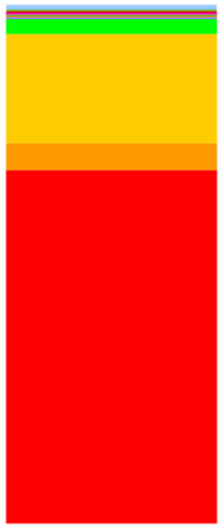

Group_HPOP1

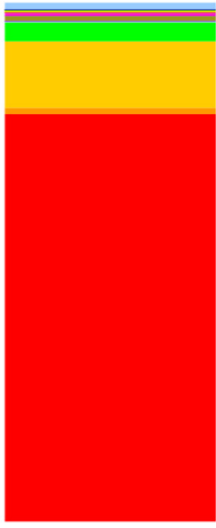

Group_LPOP1

- Lactobacillus

- Escherichia-Shigella

Streptococcus

Bifidobacteriun

- Bacteroides

Enterobacter

- Gardnerella

EClostridium sensu stricto 13

- Prevotella

-Muribaculacea

[Ruminococcus]_torques_group

Proteus

Sneathia

Enterococcus

- Atopobium

others

FIGURE 4 | Effects of POP on microbial composition of the fermentation (phylum and genus levels). Changes of microbiota composition at phylum (A) and genus (B) levels at $24 \mathrm{~h}$ in vitro fecal fermentation with POP.

$\mathrm{pH}$ value is an indicator of the rate of the fermentation reaction. Thus, POP can accelerate the speed of fermentation, which is consistent with changes in gas production in the fermentation system. At each time point, the gas production in the POP groups is significantly higher than that of the Con groups. Moreover, with the increase in polysaccharide concentration, gas production also significantly increased, which indicates that POP is a good carbon source. $\mathrm{NH}_{3}-\mathrm{N}$ is a harmful metabolite generated by 
A

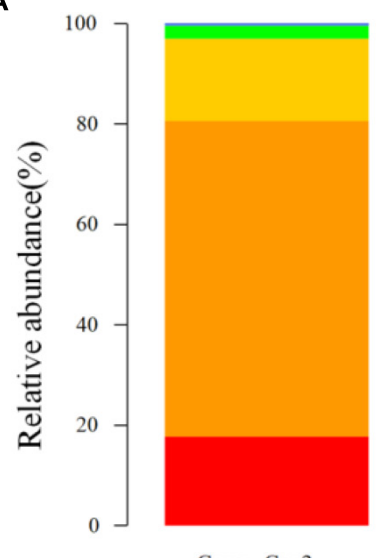

Group_Con2

-Firmicutes $=$ Proteobacteria
$=$ Bacteroidota Actinobacteriot -Fusobacteriota

- Desulfobacterot

- Acidobacteriota

Eampilobacterot

Gemmatimonadota

- Myxococcota

- Cyanobacteria

Nitrospirota

- Deferribacterota

- Latescibacterota

- Caldisericota

others

B

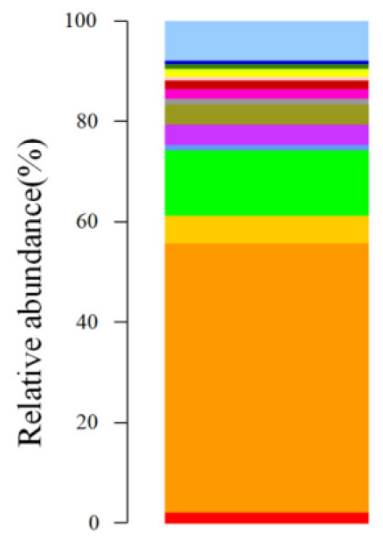

Group_Con2

- Lactobacillus Escherichia-Shigella Streptococcus

- Bacteroides

Bifidobacterium

Enterobacter

Gardnerella

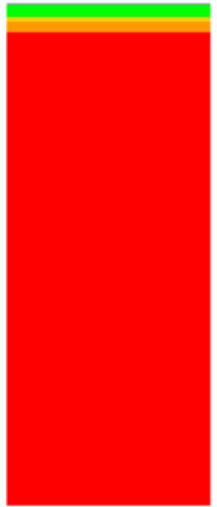

Group_HPOP2

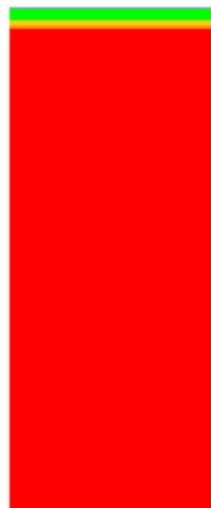

Group_LPOP2

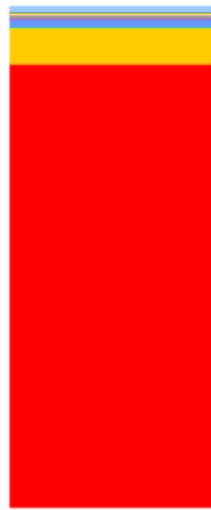

Group_LPOP2

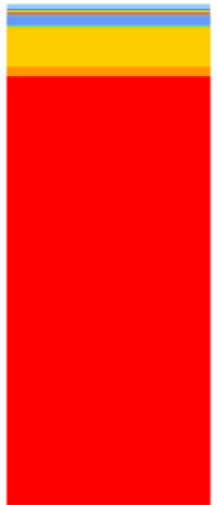

Group_HPOP2
- Parabacteroides

- Clostridium_sensu_stricto 13 Prevotella

[Ruminococcus]_torques_group

Muribaculaceae

-Clostridium_sensu_stricto_15

others

FIGURE 5 | Effects of POP on microbial composition of the fermentation (phylum and genus levels). Changes of microbiota composition at phylum (A) and genus (B) levels at $48 \mathrm{~h}$ in vitro fecal fermentation with POP.

intestinal flora. Excessive $\mathrm{NH}_{3}-\mathrm{N}$ concentration leads to an increased ammonia metabolic load or ammonia poisoning (Luo et al., 2013). We found that $\mathrm{NH}_{3}-\mathrm{N}$ levels reduced significantly in POP-treated groups, which indicates that POP has a positive role in $\mathrm{NH}_{3}-\mathrm{N}$ emissions.
Furthermore, the $\alpha$ diversity and species richness of fecal flora decreased with POP fermentation at both low and high doses. Low $\alpha$ diversity may be associated with several processes, including efficient metabolism of POP, and/or increase in the relative abundance of the intestinal flora subgroup by POP 


\section{A}

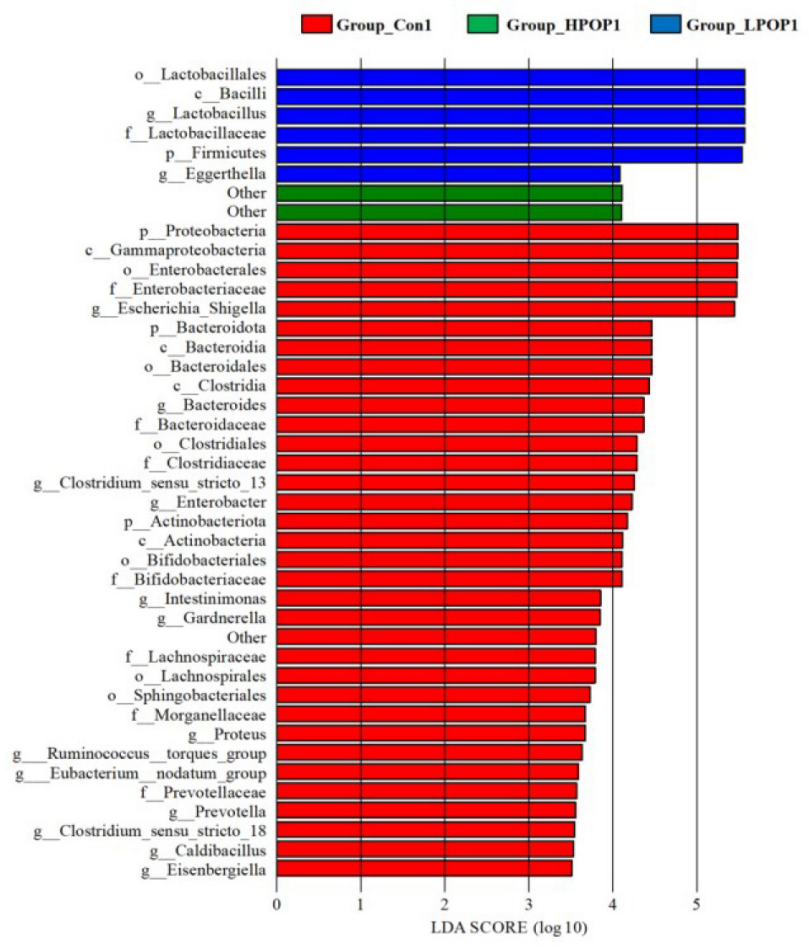

B

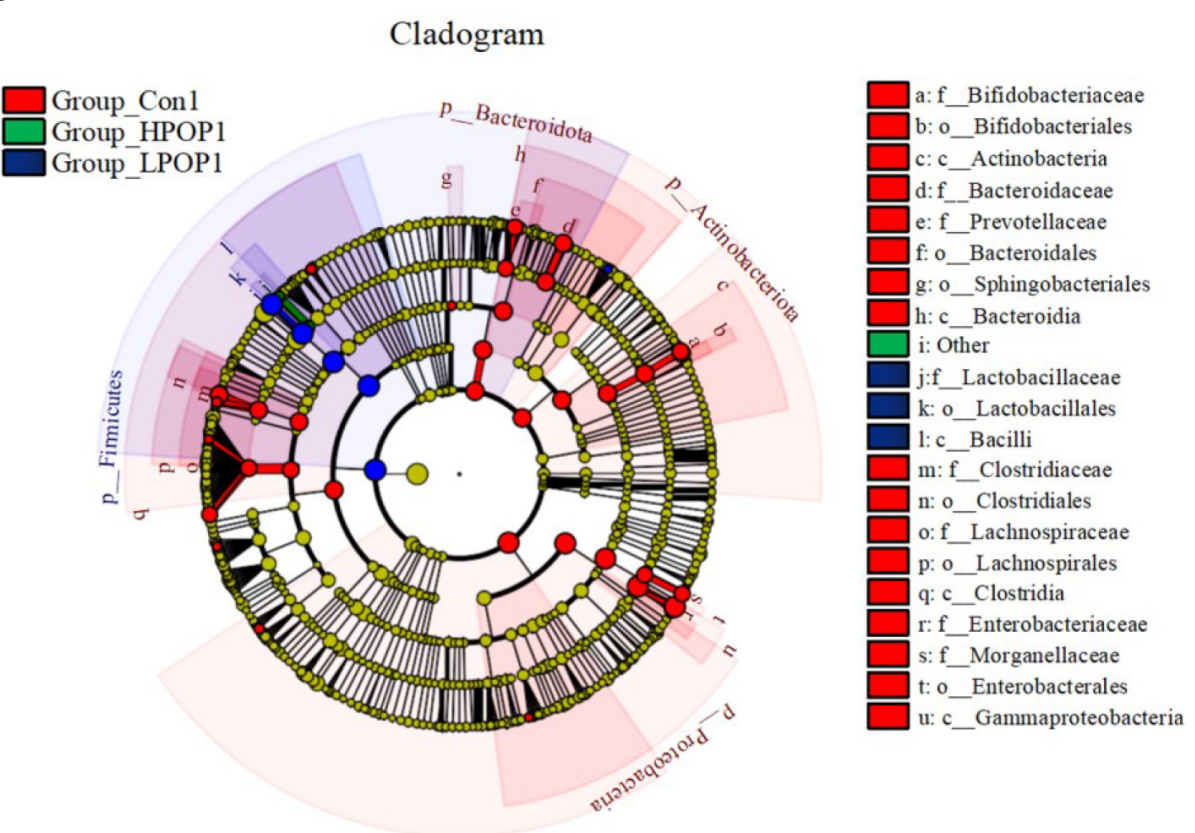

FIGURE 6 | LEfSe analysis showing the most differentially abundant taxa (LDA score $>3.0$ ) after $24 \mathrm{~h}$ in vitro fecal fermentation with POP. (A) Histogram of the LDA scores. (B) Taxonomic cladogram of all differentially abundant taxa across all taxonomic levels [from the inner to outer rings, phylum (p), class (c), order (o), family ( $f$ ), and genus (g)].

degradation products, or suppression of the growth of flora that is sensitive to a low-pH environment (Duncan et al., 2009; Bendiks et al., 2020). With the decrease in overall microbial gene richness, the level of short-chain fatty acids in the POP groups decreased significantly. Compared with the Con groups, the yields of acetic acid, butyric acid, propionic acid, and isovaleric 
A

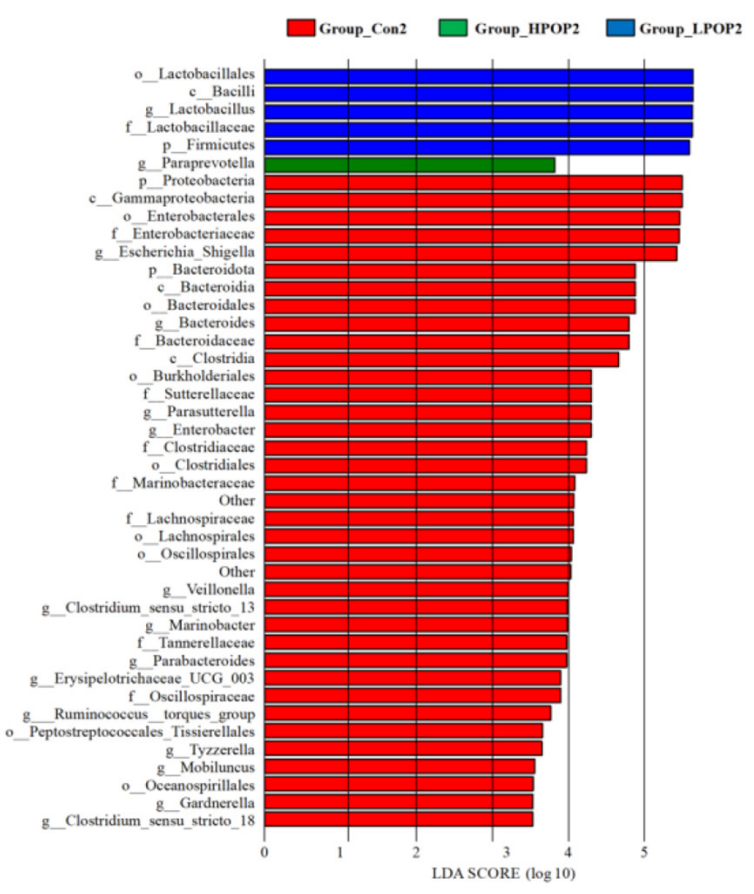

B

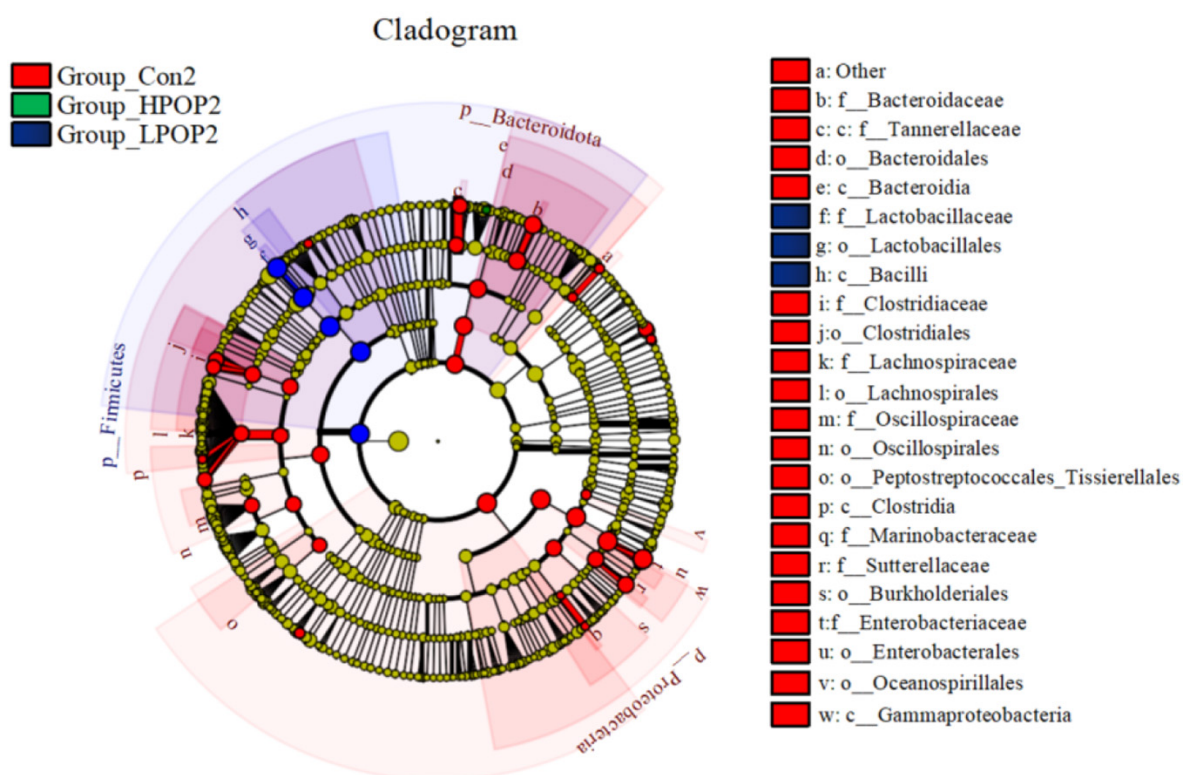

FIGURE 7 | LEfSe analysis showing the most differentially abundant taxa (LDA score $>3.0$ ) after $48 \mathrm{~h}$ in vitro fecal fermentation with POP. (A) Histogram of the LDA scores. (B) Taxonomic cladogram of all differentially abundant taxa across all taxonomic levels [from the inner to outer rings, phylum (p), class (c), order (o), family (f), and genus $(g)$.

acid in POP groups were also significantly reduced at 24 and $48 \mathrm{~h}$ of fermentation time. A recent study found that the yield of SCFAs increased significantly in the first $12 \mathrm{~h}$ of resistant starch fermentation then decreased in the following $12 \mathrm{~h}$ (Qin et al., 2021). Similar results were also observed in the in vitro fermentation of other forms of dietary fiber (Carlson et al., 2017).
Thus, we infer that the increase in fermentation time and the rapid proliferation of bacteria in the POP group result in nutrient and short-chain fatty acid consumption.

Consistent with the structure of the main flora in the human intestinal tract, the four phyla of Bacteroidetes, Firmicutes, Proteobacteria, and Actinobacteria are the most important in 


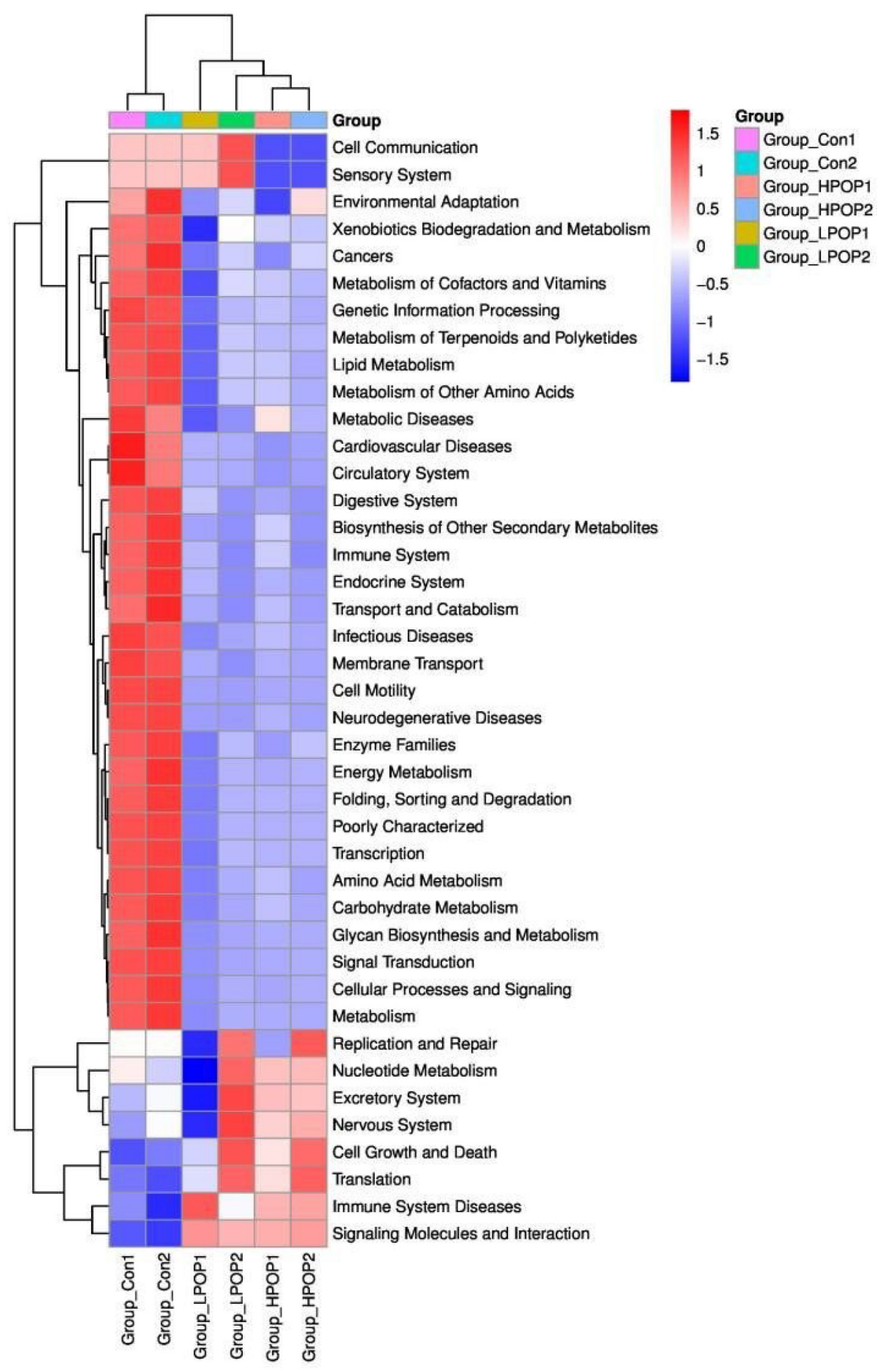

FIGURE 8 | The heatmap showing the PICRUSt-predicted KEGG ortholog (KO) functions among the Con1, Con2, LPOP1, LPOP2, HPOP1, and HPOP2 groups in in vitro fermentation.

the intestinal tract of elderly rats in this study (Simpson and Campbell, 2015; Binda et al., 2018). Firmicutes and Bacteroidetes account for more than $90 \%$ of the relative abundance of intestinal flora, and interactions between these two phyla are vital in maintaining intestinal homeostasis (Simpson and Campbell, 2015; Binda et al., 2018). Actinomycetes and Proteobacteria 
account for the remaining 10\% (Arumugam et al., 2011; Segata et al., 2012). Firmicutes are a phylum of gut microbiota and are composed of many different gram-positive bacteria. It is the main producer of butyrate in the gut and degrades indigestible polysaccharides. Bacteroidetes are mainly composed of gram-negative bacteria which are important in carbohydrate metabolism (Graf et al., 2015). Proteobacteria are composed of a variety of pathogenic bacteria, such as Escherichia coli, Salmonella, Vibrio cholerae, and Helicobacter pylori, which indicates a microecological disorder and is a potential diagnostic indicator of intestinal diseases (Shin et al., 2015; Rizzatti et al., 2017). Actinobacteria are mainly composed of gram-positive bacteria, including three major families of anaerobic bacteria (Bifidobacteria, Propionibacteria, and Corynebacteria) and one family of aerobic bacteria (Streptomyces) (Binda et al., 2018). Our present study demonstrates that POP treatment significantly promoted the relative abundance of Firmicutes while reducing the relative abundance of Bacteroidetes, Actinobacteria, and Proteobacteria, among which Proteobacteria showed the most significant decrease. These data suggest that POP can regulate the composition and function of intestinal flora by affecting the abundance of Firmicutes, which is consistent with the findings on the effects of POP on intestinal flora in weaned rats in our previous study.

To identify the specific microflora associated with POP effects, LEfSe analysis with a standard LDA score $>3.0$ was used to compare the microflora composition after 24 and $48 \mathrm{~h}$ of fermentation, to determine the group characteristics of microorganisms in each fermentative substrate. In contrast, the abundance of Lactobacillus dominated in the POP groups, while Escherichia-Shigella accounted for a high proportion in the Con groups. Lactobacillus belongs to the Firmicutes phylum, Bacilli class, and Lactobacillus family, acting as an essential regulator of homeostasis in the intestinal tract (O'Callaghan and O'Toole, 2013). It has an important role in reducing cholesterol, inhibiting the proliferation of harmful intestinal bacteria, promoting immunity, and facilitating the absorption of minerals and energy conversion (Shinohara et al., 2010; Pessione, 2012). Paraprevotella, a gram-negative anaerobe, was found to be significantly reduced in primary hypothyroidism patients (Su et al., 2020). Other studies have reported a high abundance of Paraprevotella and Eggerthella in patients with depression and an increased abundance of pro-inflammatory Escherichia-Shigella in patients with cognitive impairment and brain amyloidosis (Barandouzi et al., 2020). Likewise, our results show that POP can promote probiotic Lactobacillus in the intestinal flora of elderly rats and inhibit the reproduction of pro-inflammatory Escherichia-Shigella, which suggest that the intake of POP may be beneficial to the health of aging individuals.

The results from the PICRUSt analysis also reveal that after $48 \mathrm{~h}$ of fermentation, the proportion of genes associated with replication and repair, cell growth and death, translation, nucleotide metabolism, and excretory and nervous systems is relatively high. There is probably a connection with the increased relative abundance of Firmicutes, which metabolize starch, galactose, and butyric acid, especially with low-dose POP.
However, the expression of genes associated with infectious diseases, metabolic diseases, cardiovascular diseases, cancers, and neurodegenerative diseases in the Con groups was significantly higher than that in the POP groups, which is consistent with the results that Escherichia-Shigella was the dominant flora in the Con groups.

\section{CONCLUSION}

Firmicutes, Bacteroidetes, Proteobacteria, and Actinobacteriota are the main phyla of gut microbiota in elderly rats. However, the diversity and composition of rat gut microbiota are significantly altered after POP treatment. The intake of POP reduces the $\mathrm{pH}$ and $\mathrm{NH}_{3}-\mathrm{N}$ levels of fermentation substrates and consumes more short-chain fatty acids, which in turn promotes probiotics such as Lactobacillus and inhibits colonization of pathogenic bacteria such as Escherichia-Shigella.

This study provides new insights into the potential use of POP as a bioactive ingredient to maintain intestinal microbiota in a balanced state. Further studies should be conducted to investigate the potential physiological effects of POP fermentation on colon microecological health.

\section{DATA AVAILABILITY STATEMENT}

The datasets presented in this study can be found in online repositories. The names of the repository/repositories and accession number(s) can be found below: https://www.ncbi.nlm. nih.gov/, PRJNA789821.

\section{ETHICS STATEMENT}

The animal study was reviewed and approved by the Committee on the Ethics of Animal Experiments of the Jinggangshan University.

\section{AUTHOR CONTRIBUTIONS}

QF and XH conceived, designed the experiment, and drafted the manuscript. SZ, MY, and YL performed the experiments and collected the data. $\mathrm{GH}, \mathrm{XH}$, and $\mathrm{YH}$ provided resources and reviewed the manuscript. All authors contributed to the article and approved the submitted version.

\section{FUNDING}

This work was supported by grants from the National Natural Science Foundation of China (31860711 and 41867032), the Key Projects of Ji'an Science and Technology Bureau (JCJZ2020-83) and the Local Service Project of Jinggangshan University (JFD202108 and JFD202107), the Science and Technology Foundation of Jiangxi Education Department (GJJ211006 and GJJ201509). 


\section{REFERENCES}

Arumugam, M., Raes, J., Pelletier, E., Le Paslier, D., Yamada, T., Mende, D. R., et al. (2011). Enterotypes of the human gut microbiome. Nature 473, 174-180.

Barandouzi, Z. A., Starkweather, A. R., Henderson, W. A., Gyamfi, A., and Cong, X. S. (2020). Altered Composition of Gut Microbiota in Depression: a Systematic Review. Front. Psychiatry 11:541. doi: 10.3389/fpsyt.2020.00541

Bendiks, Z. A., Knudsen, K. E. B., Keenan, M. J., and Marco, M. L. (2020). Conserved and variable responses of the gut microbiome to resistant starch type 2. Nutr. Res. 77, 12-28. doi: 10.1016/j.nutres.2020.02.009

Binda, C., Lopetuso, L. R., Rizzatti, G., Gibiino, G., Cennamo, V., and Gasbarrini, A. (2018). Actinobacteria: a relevant minority for the maintenance of gut homeostasis. Dig. Liver Dis. 50, 421-428. doi: 10.1016/j.dld.2018.02.012

Bolger, A. M., Lohse, M., and Usadel, B. (2014). Trimmomatic: a flexible trimmer for Illumina sequence data. Bioinformatics 30, 2114-2120. doi: 10. 1093/bioinformatics/btu170

Caporaso, J. G., Kuczynski, J., Stombaugh, J., Bittinger, K., Bushman, F. D., Costello, E. K., et al. (2010). QIIME allows analysis of high-throughput community sequencing data. Nat. Methods 7, 335-336. doi: 10.1038/nmeth. f.303

Carlson, J. L., Erickson, J. M., Hess, J. M., Gould, T. J., and Slavin, J. L. (2017). Prebiotic Dietary Fiber and Gut Health: comparing the in Vitro Fermentations of Beta-Glucan. Inulin and Xylooligosaccharide. Nutrients 9:1361. doi: 10.3390/ nu9121361

Chu, C.-Q., Yu, L.-l., Chen, W., Tian, F.-W., and Zhai, Q.-X. (2021). Dietary patterns affect Parkinson's disease via the microbiota-gut-brain axis. Trends Food Sci. Technol. 116, 90-101. doi: 10.3748/wjg.v21.i37.10609

Dong, C. X., Hayashi, K., Lee, J. B., and Hayashi, T. (2010). Characterization of structures and antiviral effects of polysaccharides from Portulaca oleracea L. Chem. Pharm. Bull. 58, 507-510. doi: 10.1248/cpb.58.507

Duncan, S. H., Louis, P., Thomson, J. M., and Flint, H. J. (2009). The role of pH in determining the species composition of the human colonic microbiota. Environ. Microbiol. 11, 2112-2122. doi: 10.1111/j.1462-2920.2009.01931.x

Fang, Q., Hu, J., Nie, Q., and Nie, S. (2019). Effects of polysaccharides on glycometabolism based on gut microbiota alteration. Trends Food Sci. Technol. 92, 65-70. doi: 10.3389/fimmu.2020.588079

Ghosh, S., Dai, C., Brown, K., Rajendiran, E., Makarenko, S., Baker, J., et al. (2011). Colonic microbiota alters host susceptibility to infectious colitis by modulating inflammation, redox status, and ion transporter gene expression. Am. J. Physiol. Gastrointest. Liver. Physiol. 301, G39-G49. doi: 10.1152/ajpgi.00509. 2010

Gomaa, E. Z. (2020). Human gut microbiota/microbiome in health and diseases: a review. Antonie Van Leeuwenhoek 113, 2019-2040. doi: 10.1007/s10482-02001474-7

Graf, D., Di Cagno, R., Fak, F., Flint, H. J., Nyman, M., Saarela, M., et al. (2015). Contribution of diet to the composition of the human gut microbiota. Microb. Ecol. Health Dis. 26:26164. doi: 10.3402/mehd.v26.26164

Hu, Q., Li, Y., Liu, C., Huang, L. P., Zeng, L., Wang, S., et al. (2018). Effects of polysaccharide from Portulaca oleracea L. on voltage-gated $\mathrm{Na}(+)$ channel of INS-1 cells. Biomed. Pharmacother. 101, 572-578. doi: 10.1016/j.biopha.2018. 02.136

Huang, X., Luo, H., Huang, Y., Xu, D., Deng, X., and Wang, W. (2021). Effects of Portulacea oleracea L. Polysaccharide on Intestinal Flora in Weaned Rats. Chin. J. Anim. Nutr. 33, 4694-4707.

Iranshahy, M., Javadi, B., Iranshahi, M., Jahanbakhsh, S. P., Mahyari, S., Hassani, F. V., et al. (2017). A review of traditional uses, phytochemistry and pharmacology of Portulaca oleracea L. J. Ethnopharmacol. 205, 158-172. doi: 10.1016/j.jep.2017.05.004

Kim, C., and Kim, B. (2018). Anti-Cancer Natural Products and Their Bioactive Compounds Inducing ER Stress-Mediated Apoptosis: a Review. Nutrients 10:1021. doi: 10.3390/nu10081021

Lee, H., Lee, Y., Kim, J., An, J., Lee, S., Kong, H., et al. (2018). Modulation of the gut microbiota by metformin improves metabolic profiles in aged obese mice. Gut Microbes 9, 155-165. doi: 10.1080/19490976.2017.1405209

Luo, X., Cai, L., Ruan, Z., Huang, X., Liu, S., and OuoYang, C. (2013). Fermentation Characteristics of Four Kinds Foodstuff in Vitro. Food Res. Dev. 34, 4-8.
Mandalari, G., Nueno-Palop, C., Bisignano, G., Wickham, M. S., and Narbad, A. (2008). Potential prebiotic properties of almond (Amygdalus communis L.) seeds. Appl. Environ. Microbiol. 74, 4264-4270. doi: 10.1128/AEM.00 739-08

Mangiola, F., Nicoletti, A., Gasbarrini, A., and Ponziani, F. R. (2018). Gut microbiota and aging. Eur. Rev. Med. Pharmacol. Sci. 22, 7404-7413.

Miao, L., Tao, H., Peng, Y., Wang, S., Zhong, Z., El-Seedi, H., et al. (2019). The antiinflammatory potential of Portulaca oleracea L. (purslane) extract by partial suppression on NF-kappaB and MAPK activation. Food. Chem. 290, 239-245. doi: 10.1016/j.foodchem.2019.04.005

O'Callaghan, A., and van Sinderen, D. (2016). Bifidobacteria and Their Role as Members of the Human Gut Microbiota. Front. Microbiol. 7:925. doi: 10.3389/ fmicb.2016.00925

O'Callaghan, J., and O'Toole, P. W. (2013). Lactobacillus: host-microbe relationships. Curr. Top. Microbiol. Immunol. 358, 119-154.

Pessione, E. (2012). Lactic acid bacteria contribution to gut microbiota complexity: lights and shadows. Front. Cell. Infect. Microbiol. 2:86. doi: 10.3389/fcimb.2012. 00086

Qin, R., Wang, J., Chao, C., Yu, J., Copeland, L., Wang, S., et al. (2021). RS5 Produced More Butyric Acid through Regulating the Microbial Community of Human Gut Microbiota. J. Agric. Food Chem. 69, 3209-3218. doi: 10.1021/acs. jafc.0c08187

Reyon, D., Tsai, S. Q., Khayter, C., Foden, J. A., Sander, J. D., and Joung, J. K. (2012). FLASH assembly of TALENs for high-throughput genome editing. Nat. Biotechnol. 30, 460-465. doi: 10.1038/nbt.2170

Rizzatti, G., Lopetuso, L. R., Gibiino, G., Binda, C., and Gasbarrini, A. (2017). Proteobacteria: a Common Factor in Human Diseases. Biomed. Res. Int. 2017:9351507. doi: 10.1155/2017/9351507

Rognes, T., Flouri, T., Nichols, B., Quince, C., and Mahe, F. (2016). VSEARCH: a versatile open source tool for metagenomics. PeerJ. 4:e2584. doi: 10.7717/peerj. 2584

Salazar, N., Gonzalez, S., Nogacka, A. M., Rios-Covian, D., Arboleya, S., Gueimonde, M., et al. (2020). Microbiome: effects of Ageing and Diet. Curr. Issues Mol. Biol. 36, 33-62. doi: 10.21775/cimb.036.033

Segata, N., Haake, S. K., Mannon, P., Lemon, K. P., Waldron, L., Gevers, D., et al. (2012). Composition of the adult digestive tract bacterial microbiome based on seven mouth surfaces, tonsils, throat and stool samples. Genome Biol. 13:R42. doi: 10.1186/gb-2012-13-6-r42

Shin, N. R., Whon, T. W., and Bae, J. W. (2015). Proteobacteria: microbial signature of dysbiosis in gut microbiota. Trends Biotechnol. 33, 496-503. doi: 10.1016/j. tibtech.2015.06.011

Shinohara, K., Ohashi, Y., Kawasumi, K., Terada, A., and Fujisawa, T. (2010). Effect of apple intake on fecal microbiota and metabolites in humans. Anaerobe 16, 510-515. doi: 10.1016/j.anaerobe.2010.03.005

Simpson, H. L., and Campbell, B. J. (2015). Review article: dietary fibre-microbiota interactions. Aliment Pharmacol. Ther. 42, 158-179. doi: 10.1111/apt.1 3248

Su, X., Zhao, Y., Li, Y., Ma, S., and Wang, Z. (2020). Gut dysbiosis is associated with primary hypothyroidism with interaction on gut-thyroid axis. Clin. Sci. 134, 1521-1535. doi: 10.1042/CS20200475

Vemuri, R. C., Gundamaraju, R., Shinde, T., and Eri, R. (2017). Therapeutic interventions for gut dysbiosis and related disorders in the elderly: antibiotics, probiotics or faecal microbiota transplantation? Benef. Microbes 8, 179-192. doi: 10.3920/BM2016.0115

Wang, H., Zhang, X., Wang, S., Li, H., Lu, Z., Shi, J., et al. (2018). Mannanoligosaccharide modulates the obesity and gut microbiota in high-fat diet-fed mice. Food Funct. 9, 3916-3929. doi: 10.1039/c8fo00209f

Wang, M., Chen, Y., Wang, Y., Li, Y., Zheng, H., Ma, F., et al. (2018). The effect of probiotics and polysaccharides on the gut microbiota composition and function of weaned rats. Food Funct. 9, 1864-1877. doi: 10.1039/c7fo01507k

Wang, Q., Garrity, G. M., Tiedje, J. M., and Cole, J. R. (2007). Naive Bayesian classifier for rapid assignment of rRNA sequences into the new bacterial taxonomy. Appl. Environ. Microbiol. 73, 5261-5267. doi: 10.1128/AEM.00 062-07

Xu, L., Li, Y., Dai, Y., and Peng, J. (2018). Natural products for the treatment of type 2 diabetes mellitus: pharmacology and mechanisms. Pharmacol. Res. 130, 451-465. doi: 10.1016/j.phrs.2018.01.015 
Yu, M., Jia, H. M., Zhou, C., Yang, Y., Sun, L. L., and Zou, Z. M. (2017). Urinary and Fecal Metabonomics Study of the Protective Effect of Chaihu-Shu-GanSan on Antibiotic-Induced Gut Microbiota Dysbiosis in Rats. Sci. Rep. 7:46551. doi: 10.1038/srep46551

Zhou, B., Yuan, Y., Zhang, S., Guo, C., Li, X., Li, G., et al. (2020). Intestinal Flora and Disease Mutually Shape the Regional Immune System in the Intestinal Tract. Front. Immunol. 11:575. doi: 10.3389/fimmu.2020.00575

Zhou, Y. X., Xin, H. L., Rahman, K., Wang, S. J., Peng, C., and Zhang, H. (2015). Portulaca oleracea L.: a review of phytochemistry and pharmacological effects. Biomed. Res. Int. 2015:925631. doi: 10.1155/2015/925631

Zhu, K., Fan, H., Zeng, S., Nie, S., Zhang, Y., Tan, L., et al. (2021). Polysaccharide from Artocarpus heterophyllus Lam. (jackfruit) pulp modulates gut microbiota composition and improves short-chain fatty acids production. Food Chem. 364:130434. doi: 10.1016/j.foodchem.2021.130434

Zimmer, J., Lange, B., Frick, J. S., Sauer, H., Zimmermann, K., Schwiertz, A., et al. (2012). A vegan or vegetarian diet substantially alters the human colonic faecal microbiota. Eur. J. Clin. Nutr. 66, 53-60. doi: 10.1038/ejcn.2011.141
Conflict of Interest: The authors declare that the research was conducted in the absence of any commercial or financial relationships that could be construed as a potential conflict of interest.

Publisher's Note: All claims expressed in this article are solely those of the authors and do not necessarily represent those of their affiliated organizations, or those of the publisher, the editors and the reviewers. Any product that may be evaluated in this article, or claim that may be made by its manufacturer, is not guaranteed or endorsed by the publisher.

Copyright (c) $2022 \mathrm{Fu}, \mathrm{Zhou}, \mathrm{Yu}, \mathrm{Lu}, \mathrm{He}$, Huang and Huang. This is an openaccess article distributed under the terms of the Creative Commons Attribution License (CC BY). The use, distribution or reproduction in other forums is permitted, provided the original author(s) and the copyright owner(s) are credited and that the original publication in this journal is cited, in accordance with accepted academic practice. No use, distribution or reproduction is permitted which does not comply with these terms. 